\title{
MEDICIÓN DE LA EFICIENCIA Y PRODUCTIVIDAD DE LOS COLEGIOS OFICIALES DE BARRANQUILLA
}

Adel Alfonso Mendoza Mendoza ${ }^{1}$, Leidys Romero Fontalvo ${ }^{2}$, Elkin Julio Blanco ${ }^{3}$

\section{Resumen}

Introducción. En este artículo se presenta una metodología que permite que los colegios públicos (también llamados oficiales) de la ciudad de Barranquilla evalúen su desempeño respecto a otras instituciones oficiales y establezcan planes de mejoras potenciales para su organización. Objetivo. Evaluar y analizar, mediante la técnica de análisis envolvente de datos (DEA), la eficiencia relativa y la productividad de acuerdo con el índice de Malmquist en el uso de los recursos de los colegios oficiales del distrito de Barranquilla. Materiales y métodos. Se identificaron las variables de entradas y salidas mediante la metodología multicriterio y la aplicación de encuestas a expertos y se siguieron los lineamientos propuestos por Golany y Roll (1) para la utilización de la técnica DEA. Resultados. Los resultados de este estudio indican que, de los 148 colegios evaluados, 56 presentaron un incremento en su productividad, es decir, el 38\% del total de las instituciones. Así mismo, se observó que en los años 2012 y 2013 el número de colegios eficientes fueron 54 y 57, respectivamente. Conclusión. Se evaluó la eficiencia considerando el modelo DEA CCR-O con retornos a escala constantes. El análisis de la eficiencia se efectuó teniendo en cuenta las cinco localidades del distrito de Barranquilla (Riomar, Norte-Centro Histórico, Metropolitana, Suroriente y Suroccidente) y se generaron los valores de eficiencia para los años 2012 y 2013.

Palabras clave: productividad, análisis envolvente de datos, índice de Malmquist, eficiencia.

\footnotetext{
${ }^{1}$ Magíster en Ingeniería Industrial. Profesor de Tiempo Completo de la Universidad del Atlántico. Grupo de Investigación3i+d. Email: adelmendoza@uniatlantico. edu.co

${ }^{2}$ Ingeniera Industrial de la Universidad del Atlántico.

${ }^{3}$ Ingeniero Industrial de la Universidad del Atlántico.
} 


\section{MEASUREMENT OF THE EFFICIENCY AND PRODUCTIVITY OF THE PUBLIC SCHOOLS OF BARRANQUILLA}

Adel Alfonso Mendoza Mendoza ${ }^{1}$, Leidys Romero Fontalvo ${ }^{2}$, Elkin Julio Blanco ${ }^{3}$

\section{Abstract}

Introduction. In this article is shown a methodology that allows each of the public schools of the city of Barranquilla to assses their performance with respect to other official institutions, providing them with the opportunity to establish plans for potential improvements for their organization. Objective. To evaluate and analyze through the Data Envelopment Analysis technique (DEA in Spanish), the relative efficiency and productivity with the Malmquist index regarding the use of resources of the public schools of the District of Barranquilla. Materials and methods. Identification of input and output variables using Multicriteria methodology and application of surveys to experts, following the guidelines proposed by Golany and Roll for the use of the DEA technique. Results. The results of this study indicate that, from the 148 schools analyzed, 56 had an increase in their productivity, that is, $38 \%$ of the total number of institutions. Likewise, it was observed that in the years2012 and 2013 the number of efficient schools are 54 and 57 respectively. Conclusion. The efficiency was evaluated considering the DEA CCR-O model with constant returns to scale. The efficiency analysis was carried out taking into account the five localities of the Barranquilla District (Riomar, Norte Centro Histórico, Metropolitana, Suroriente and Suroccidente), and efficiency values were generated for the years 2012 and 2013. 


\section{MEDIÇÃO DA EFIIIÊNCIA E PRODUTIVIDADE DAS ESCOLAS OFICIAIS DE BARRANQUILLA}

Adel Alfonso Mendoza Mendoza ${ }^{1}$, Leidys Romero Fontalvo ${ }^{2}$, Elkin Julio Blanco ${ }^{3}$

\section{Resumo}

Introdução. Este artigo apresenta uma metodologia que permite que cada uma das escolas oficiais da cidade de Barranquilla avalie seu desempenhoem relaçãoàs outrasinstituições oficiais, proporcionandolhes a oportunidade de estabelecer planos de melhorias potenciais para sua organização. Objetivo. Avaliar e analisar mediante a técnica de análise por envoltória de dados (DEA), a eficiência relativa e a produtividade com o índice de Malmquist no uso dos recursos das escolas oficiais do Distrito de Barranquilla. Materiais e métodos. Identificação de variáveis de entrada e saída usando metodologia multicritério e aplicação de pesquisas a especialistas e seguindo as diretrizes propostas por Golany e Roll para o uso da técnica DEA. Resultados. Os resultados deste estudo indicam que, das 148 escolas avaliadas, 56 tiveram aumento de produtividade, ou seja, 38\% do total de instituições. Da mesma forma, observou-se que nos anos de 2012 e 2013 o número de escolas eficientes é de 54 e 57, respectivamente. Conclusão. A eficiência foi avaliada considerando o modelo DEA CCR-O com retornos constantes de escala. A análise de eficiência foi feita tendo em conta as cinco localidades no Distrito de Barranquilla (Riomar, Norte Centro Histórico, Metropolitana, Suroriente e Suroccidente) e valores de eficiência para os anos de 2012 e 2013 foram gerados. 


\section{Introducción}

El sistema educativo en Colombia se conforman por los niveles de educación inicial, la educación preescolar, la educación básica, que incluye el ciclo primario y secundario, la educación media, y la educación superior(2). Según lo establecido por la ley 115 de 1994 o Ley General de Educación, y según lo dispuesto en la carta magna de Colombia, en Colombia la educación es un derecho y un servicio público. Sin embargo, la inversión en educación en el país no llega al 5\% del PIB, de acuerdo con la información registrada en los datos del Banco Mundial(3), en el periodo entre 2009 y 2013. Por tal motivo, se hace necesaria la buena asignación de estos recursos para que la posibilidad de acceder a un sistema de calidad no se dificulte ni se generen deficiencias en la cobertura.

En los últimos años, se observa que en el distrito de Barranquilla se ha gestionado la apertura de nuevos centros oficiales de formación, lo que representa un avance en términos de cobertura educativa; sin embargo, esto no es garantía de mayores avances en términos de calidad educativa. Así mismo, el gobierno ha implementado el índice sintético de calidad educativa, el cual mide los resultados de cada institución educativa cada año, pero esto no permite establecer si los recursos destinados a la educación se están utilizando eficientemente, o si el monto actual de estos recursos es suficiente para lograr niveles óptimos de calidad. De aquí surge la necesidad de evaluar el desempeño actual del sistema educativo que abarca los centros oficiales del distrito de Barranquilla.
En este trabajo de investigación se determinan la eficiencia y la productividad de los colegios oficiales de Barranquilla durante el periodo entre los años 2012 y 2013. Para ello, se utiliza el análisis envolvente de datos (DEA) y el índice de Malmquist. Actualmente, cada vez es mayor la atención a la gestión de recursos públicos, razón por la cual surge la necesidad de evaluar el desempeño de los colegios del distrito de Barranquilla a través de una herramienta que pueda proporcionarnos resultados confiables que permitan plantear mejoras en la organización y gestión de cada plantel educativo.

\section{Metodología}

La metodología del análisis envolvente de datos (DEA) fue desarrollada por Charnes, Cooper y Rhodes, basados los conceptos concebidos por Farrel en 1957(4). Es una técnica de programación lineal que se utiliza para comparar unidades de producción que manejan el mismo grupo de recursos y producen el mismo grupo de productos, generando así una frontera eficiente e indicadores relativos de eficiencia dentro de la población de unidades de producción bajo estudio. Es así como las instituciones educativas oficiales de Barranquilla se pueden tomar como una industria que transforma recursos o insumos en productos. Cada institución educativa, tratada como una DMU (Decision Making Unit), puede ser vista como una firma multi-producto(5), es decir, cada institución educativa cuenta con unos recursos (personal docente, personal administrativo y recursos físicos) para lleva a cabo su función misional y a partir de 
estos recursos obtener unos productos que se pueden medir a través del desempeño de cada institución en las pruebas de estado.

El DEA es una de las principales técnicas usadas en el sector público y privado para evaluar el desempeño de unidades productivas. Su uso es tan amplio que se pueden citar, entre algunas de sus aplicaciones, las realizadas en la evaluación de eficiencias en bancos(6), en la evaluación de riesgos crediticios(7) y en la evaluación del desempeño en el sector de la salud(8). La técnica DEA también ha sido usada en evaluación del desempeño en instituciones de educación como en la investigación de $\operatorname{Sav}(9)$ en donde se compara la eficiencia operativa de colegios en Estados Unidos. Nazarko y Šaparauskas(10) evaluaron la eficiencia de universidades tecnológicas polacas, Liu et al(11) estimaron la eficiencia técnica de escuelas secundarias y preparatorias en Taipéi (China) y Garzón et al(12) analizaron la eficiencia técnica de instituciones públicas de educación secundaria del estado Barinas (Venezuela). En Colombia se encuentran los estudios realizados por: Quesada et al (13), quienes analizaron la cobertura educativa de los municipios en el departamento de Bolívar; Iregui et al(14), que determinaron la eficiencia técnica de colegios públicos y privados del país; $\mathrm{y}$ Soto et al(15), que efectuaron un estudio para calcular y analizar la eficiencia relativa en cobertura y calidad de las instituciones educativas del municipio de Dos Quebradas, Risaralda.

El índice de la variación de productividad Malmquist fue introducido por
Caves et al(16) en 1982, inspirado en el trabajo de Malmquist(17). Su objetivo es medir la variación de la productividad en dos periodos de tiempo. Supóngase que, para una DMU0, se evalúa la eficiencia para dos periodos de tiempo $t$ y $\mathrm{t}+1$ y que existe una función de producción para cada periodo de tiempo evaluado. El índice de productividad de Malmquist, orientado a los inputs, mide la variación de productividad para la DMU0 entre los periodos $t$ y $t+1$. Dentro de las aplicaciones del índice de Malmquist en el sector educativo podemos destacar algunas investigaciones como: Aparicio et al(18), Podinovski et al(19) y Essid et al(20), entre otros.

\section{Materiales y métodos}

La presente investigación de naturaleza cuantitativa es un estudio empírico soportado en el análisis envolvente de datos (DEA) que consiste en determinar los valores de eficiencia técnica de 148 de las 152 instituciones educativas distritales (IED) de Barranquilla durante los años 2012 y 2013, mediante el modelo DEA-CCR con enfoque en salidas. Con base en estos resultados se determinó el índice de productividad de Malmquist para cada IED. En el estudio se excluyeron 4 de las 152 Instituciones debido a la falta de la información requerida. El modelo DEA-CCR también se puede usar para determinar si los rendimientos a escala están aumentando o disminuyendo(21).

El desarrollo de esta investigación se llevó a cabo según los lineamientos propuestos por puestos por Golany y Roll 
(1) para la utilización de la técnica DEA, en los que plantean las siguientes tres fases:

- Definición y selección de cada una de las DMU que van a ser incluidas en el análisis.

- Aplicación del modelo DEA CCR.

- Cálculo del índice de productividad de Malmquist.

Para la identificación de las variables se consultaron otras investigaciones que se tomaron como guía, y a partir de allí se seleccionaron las variables a utilizar en el modelo DEA-CCR, empleando la metodología de juicio de expertos pro- puesta por Escobar y Cuervo (22), con el propósito de disminuir el número de variables de entradas y salidas del análisis de eficiencia y, de esta forma, mejorar el poder discriminatorio del DEA (23). Se consultaron diez expertos: cuatro directivos de planteles educativos oficiales, cuatro doctores en educación de la Facultad de Ciencias de la Educación de la Universidad Del Atlántico y dos funcionarios de la Secretaría de Educación del distrito de Barranquilla. Los expertos realizaron una valoración de las diferentes variables de estudios realizados por otros autores y a partir de esta valorización se realizó la selección de variables a través de una evaluación multicriterio. A continuación, en la Tabla 1, se muestran las variables incluidas en el estudio.

Tabla 1. Variables incluidas en el estudio

\begin{tabular}{|c|c|}
\hline Variables de entrada & Variables de salida \\
\hline 1. Matrícula oficial del plantel educativo & $\begin{array}{l}\text { 1. Número de alumnos que alcanzan los logros } \\
\text { en el año escolar }\end{array}$ \\
\hline 2. Nómina del personal docente anual & 2. NO deserción en la IE (permanencia) \\
\hline 3. Nómina de personal administrativo & $\begin{array}{l}\text { 3. Promedio de las competencias en matemáti- } \\
\text { cas SABER } 11\end{array}$ \\
\hline $\begin{array}{l}\text { 4. Número de aulas de clase del plantel } \\
\text { educativo }\end{array}$ & $\begin{array}{l}\text { 4. Promedio de las competencias en ciencias } \\
\text { naturales SABER } 11\end{array}$ \\
\hline 5. Metros cuadrados construidos & $\begin{array}{l}\text { 5. Promedio de las competencias en humanida- } \\
\text { des SABER } 11\end{array}$ \\
\hline 6. Número de computadores & $\begin{array}{l}\text { 6. Promedio de las competencias en inglés } \\
\text { SABER } 11\end{array}$ \\
\hline & $\begin{array}{l}\text { 7. Promedio de las competencias en lenguaje } \\
\text { SABER } 11\end{array}$ \\
\hline
\end{tabular}

Fuente: Elaboración propia de los autores.

Para llevar a cabo el proceso de medi14 ción de las variables de entrada y de salida, se definió el conjunto de institucio- nes educativas oficiales que formarían parte del estudio. De los 152 colegios públicos con los que cuenta el distrito de 
Barranquilla, se tuvieron en cuenta 148. Dado que en las bases de datos consultadas no había información disponible para estas instituciones, se excluyeron del estudio a las siguientes: Centro de Educación Básica \# 201, Centro de Educación Básica Etnoeducativo Paulino Salgado "Batata", IED Angelina De Montes e IED Niño Jesús.

La búsqueda de información de las variables de entrada y de salida para cada IED se realizó para los años 2012 y 2013 , ya que el objetivo era evaluar el cambio que se produce en la eficiencia técnica según los cambios tecnológicos presentados durante este periodo de tiempo. La información con la que se efectuó el análisis fue recolectada de varias fuentes, entre las cuales se encuentran las bases de datos de la Secretaría de Educación de Barranquilla (SED), de la que se destaca el Sistema de Información Nacional de Educación Básica y Media (SINEB)(24), a través del cual los establecimientos educativos deben reportar a la SED la información referente a la matrícula oficial y no oficial, la nómina, la infraestructura física y tecnológica entre otras. Se consultaron además las bases de datos del Instituto Colombiano para el Fomento de la Educación Superior (ICFES) y los registros de algunas IED que no tenían reportada la información solicitada a la SED.

Los datos de las variables de entrada (matrícula oficial, número de aulas de clase, metros cuadrados de área construida, número de computadores) así como la información para determinar los valores de las variables de salida (número de alumnos que alcanzan los logros y no deserción o permanencia) fueron tomados de las bases de datos de la SED y de las visitas a las instituciones educativas. Los valores de las nóminas anuales de personal docente $y$ administrativo se determinaron teniendo en cuenta la asignación salarial para el escalafón de la planta docente de cada IED, como lo reglamenta el Departamento Administrativo de la Función Pública a través de los Decretos 1055 y 1027 del 4 de abril de 2011(25,26), por medio de los cuales se modifican las remuneraciones de los servidores públicos docentes y directivos docentes. Se manejaron pesos constantes para cada año.

Los resultados obtenidos por los establecimientos educativos en las distintas competencias (matemáticas; ciencias naturales, integrada por química, física y biología; humanidades, integrada por ciencias sociales y filosofía; lenguaje e inglés), se obtuvieron de las estadísticas manejadas por el ICFES en sus bases de datos.

\section{Resultados}

Como se mencionó anteriormente, la determinación de los valores de eficiencia se realizó mediante el modelo DEACCR. Cabe resaltar que la mayor parte de las contribuciones en DEA son hechas por dos modelos estándar: el CCR y el BCC (27). El modelo CCR calcula las eficiencias bajo la hipótesis de retornos constantes a escala, es decir, compara unidades homogéneas; el modelo BBC calcula las eficiencias con retornos variables a escala en donde cada unidad ineficiente solo se compara con una uni- 
dad eficiente, pero de sus mismas características. De acuerdo con la literatura de la técnica DEA, se emplearon "rendimientos constantes a escala" para caracterizar el modelo CCR, esto es, técnicamente, correcto, pero algo e puede usar para determinar si los rendimientos a escala están aumentando o disminuyendo (21). Como se ha descrito anterior- mente en este artículo de investigación, la eficiencia de las instituciones educativas del distrito de Barranquilla consideradas objeto de estudio será evaluada mediante el modelo DEA CCR-O. En la Tabla 2 y Tabla 3 se muestra el resumen de los principales estadísticos descriptivos para las variables de entrada y de salida, respectivamente.

Tabla 2. Estadísticos descriptivos de las variables de entrada

\begin{tabular}{|c|c|c|c|c|c|c|}
\hline \multicolumn{2}{|c|}{ Matrícula oficial } & $\begin{array}{l}\text { Valor de la } \\
\text { nómina do- } \\
\text { cente anual }\end{array}$ & $\begin{array}{l}\text { Nómina del } \\
\text { personal admi- } \\
\text { nistrativo }\end{array}$ & $\begin{array}{c}\text { Número de } \\
\text { aulas de } \\
\text { clase }\end{array}$ & $\begin{array}{c}\mathrm{M}^{2} \\
\text { cons- } \\
\text { truidos }\end{array}$ & $\begin{array}{c}\text { Número de } \\
\text { computa- } \\
\text { dores }\end{array}$ \\
\hline \multicolumn{7}{|l|}{2012} \\
\hline Media & 1397 & 87512291 & 92625517 & 18 & 1661 & 36 \\
\hline Mediana & 1251 & 742874359 & 83904215 & 16 & 1412 & 29 \\
\hline $\begin{array}{l}\text { Desv. } \\
\text { Estándar }\end{array}$ & 1620 & 1753107659 & 134366304 & 28 & 3859 & 134 \\
\hline \multicolumn{7}{|l|}{2013} \\
\hline Media & 1273 & 909725768 & 93152486 & 19 & 1965 & 38 \\
\hline Mediana & 1160 & 803022336 & 84538210 & 17 & 1412 & 33 \\
\hline $\begin{array}{l}\text { Desv. } \\
\text { Estándar }\end{array}$ & 1394 & 1753107659 & 134366304 & 28 & 3859 & 134 \\
\hline
\end{tabular}

Fuente. Elaboración propia de los autores

Tabla 3. Estadísticos descriptivos de las variables de entrada

\begin{tabular}{|c|c|c|c|c|c|c|c|}
\hline & $\begin{array}{l}\text { PROM } \\
\text { Mate- } \\
\text { máticas } \\
\text { SABER } \\
11\end{array}$ & $\begin{array}{c}\text { PROM } \\
\text { Ciencias } \\
\text { Naturales } \\
\text { SABER } 11\end{array}$ & $\begin{array}{c}\text { PROM } \\
\text { Huma- } \\
\text { nidades } \\
\text { SABER } 11\end{array}$ & $\begin{array}{l}\text { PROM } \\
\text { Inglés } \\
\text { SABER } \\
11\end{array}$ & $\begin{array}{l}\text { PROM } \\
\text { Len- } \\
\text { guaje } \\
\text { SABER } \\
11\end{array}$ & $\begin{array}{l}\text { Número } \\
\text { de alum- } \\
\text { nos que } \\
\text { alcanzan } \\
\text { los logros }\end{array}$ & $\begin{array}{c}\text { NO } \\
\text { deser- } \\
\text { ción }\end{array}$ \\
\hline
\end{tabular}

$$
2012
$$

\begin{tabular}{|c|c|c|c|c|c|c|c|}
\hline Media & 50 & 50 & 46 & 48 & 50 & 1287 & 1370 \\
\hline Mediana & 47 & 46 & 42 & 44 & 47 & 1155 & 1222 \\
\hline $\begin{array}{c}\text { Desv. } \\
\text { Estándar }\end{array}$ & 8 & 4 & 5 & 2 & 7 & 1413 & 1613 \\
\hline
\end{tabular}


Continuación tabla 3. Estadísticos descriptivos de las variables de salida.

\begin{tabular}{|c|c|c|c|c|c|c|c|}
\hline & $\begin{array}{l}\text { PROM } \\
\text { Mate- } \\
\text { máticas } \\
\text { SABER } \\
11\end{array}$ & $\begin{array}{c}\text { PROM } \\
\text { Ciencias } \\
\text { Naturales } \\
\text { SABER } 11\end{array}$ & $\begin{array}{c}\text { PROM } \\
\text { Huma- } \\
\text { nidades } \\
\text { SABER } 11\end{array}$ & $\begin{array}{l}\text { PROM } \\
\text { Inglés } \\
\text { SABER } \\
11\end{array}$ & $\begin{array}{c}\text { PROM } \\
\text { Len- } \\
\text { guaje } \\
\text { SABER } \\
11\end{array}$ & $\begin{array}{l}\text { Número } \\
\text { de alum- } \\
\text { nos que } \\
\text { alcanzan } \\
\text { los logros }\end{array}$ & $\begin{array}{c}\text { NO } \\
\text { deser- } \\
\text { ción }\end{array}$ \\
\hline \multicolumn{8}{|l|}{2013} \\
\hline Media & 49 & 49 & 46 & 47 & 50 & 1142 & 1244 \\
\hline Mediana & 46 & 46 & 43 & 44 & 47 & 1024 & 1104 \\
\hline $\begin{array}{c}\text { Desv. } \\
\text { Estándar }\end{array}$ & 2 & 3 & 5 & 4 & 3 & 1273 & 1411 \\
\hline
\end{tabular}

Fuente. Elaboración propia de los autores

La medición de la productividad en este trabajo se realizó utilizando el índice de productividad de Malmquist (IPM), basado en la metodología DEA, propuesto por Färe et al (28) que descompone el
IPM en dos componentes: cambio de la eficiencia técnica y cambio de la frontera tecnológica de la DMU0. La multiplicación de estos cambios da como resultado el índice de productividad.

$$
I P M_{0} \frac{D_{0}^{\mathrm{t}+1}\left(x^{\mathrm{t}+1}, y^{\mathrm{t}+1}\right)}{D_{0}^{\mathrm{t}}\left(x^{\mathrm{t}}, y^{\mathrm{t}}\right)}\left[\frac{D_{0}^{\mathrm{t}}\left(x^{\mathrm{t}+1}, y^{\mathrm{t}+1}\right) D_{0}^{\mathrm{t}}\left(x^{\mathrm{t}}, y^{\mathrm{t}}\right)}{D_{0}^{\mathrm{t}+1}\left(x^{\mathrm{t}+1}, y^{\mathrm{t}+1}\right) D_{0}^{\mathrm{t}+1}\left(x^{\mathrm{t}}, y^{\mathrm{t}}\right)}\right]^{1 / 2}
$$

En donde el primer término, cambio de la eficiencia técnica, refleja los cambios en la eficiencia relativa de una unidad i. El segundo término, el cambio tecnológico, mide el cambio en la propia frontera de producción y refleja los efectos que se refieren al sistema de IED en su conjunto (29).
En la Tabla 4 se muestran los resultados de eficiencia para los años 2012 y 2013, asimismo, los índices de productividad para cada una de las instituciones educativas estudiadas durante este periodo. 
INVESTIGACIONES ANDINA No. 37, Vol. 20

Tabla 4. Eficiencia e Índice de Productividad de Malmquist.

\begin{tabular}{|c|c|c|c|}
\hline Institución Educativa & $\begin{array}{l}\text { Eficiencia } \\
\text { CCR } 2012\end{array}$ & $\begin{array}{l}\text { Eficiencia } \\
\text { CCR } 2013\end{array}$ & $\begin{array}{l}\text { Índice De } \\
\text { Productividad }\end{array}$ \\
\hline C.C.E.B.\# 187 CÍVICO 7 DE ABRIL & 1,0000 & 1,0000 & 0,934 \\
\hline CENT. EDUC. DIST. \# 062 & 0,9597 & 0,9851 & 1,005 \\
\hline C.C.E.B. Y MEDIA \# 197 & 1,0000 & 0,9303 & 0,883 \\
\hline C.E.B. Y MEDIA \# 103 & 1,0000 & 1,0000 & 0,972 \\
\hline C.E.B. Y MEDIA \# 132 & 1,0000 & 1,0000 & 1,062 \\
\hline C.E.B. Y MEDIA \# 161 & 0,9861 & 1,0000 & 0,980 \\
\hline C.E.B. Y MEDIA \# 176 & 0,9732 & 0,9808 & 1,000 \\
\hline C.E.B. SALVADOR ENTREGAS & 1,0000 & 1,0000 & 0,846 \\
\hline C.E.D. JESUS MAESTRO FÉ Y ALEGRÍA & 0,9856 & 0,9907 & 1,005 \\
\hline COL. DIST. ALFONSO LÓPEZ & 0,8162 & 0,7939 & 0,968 \\
\hline COL. DIST. ESTHER DE PELAEZ & 1,0000 & 1,0000 & 1,027 \\
\hline COL. COM. DIST. VILLA DEL CARMEN & 0,9765 & 0,9226 & 0,944 \\
\hline COL. COM. DIST. PABLO NERUDA & 0,9764 & 0,9592 & 0,978 \\
\hline COL. DE BARRANQUILLA CODEBA & 0,9958 & 0,9081 & 0,904 \\
\hline COL. DIST. COSTA CARIBE & 1,0000 & 1,0000 & 0,995 \\
\hline COL. DIST. DE BACHILLERATO SAN LUIS & 1,0000 & 1,0000 & 0,943 \\
\hline COL. DIST. GABRIEL GARCÍA MÁRQUEZ & 0,9903 & 1,0000 & 1,002 \\
\hline $\begin{array}{l}\text { COL. DIST. DE EDUC. COOP. Y PARA EL } \\
\text { TRABAJO }\end{array}$ & 1,0000 & 1,0000 & 0,966 \\
\hline COL. DIST. EL SILENCIO & 0,9900 & 0,9655 & 0,975 \\
\hline COL. DIST. LOS ROSALES & 0,9132 & 0,9339 & 1,023 \\
\hline COL. DIST. MARÍA AUXILIADORA & 0,9954 & 0,9642 & 0,952 \\
\hline COL. DIST. MARÍA INMACULADA & 0,9853 & 0,9831 & 0,998 \\
\hline COL. DIST. MARIE POUSSEPIN & 1,0000 & 1,0000 & 1,000 \\
\hline COL. DIST. SARID ARTETA DE VÁSQUEZ & 0,9029 & 0,9137 & 1,010 \\
\hline COL. DIST. BOSTON & 1,0000 & 1,0000 & 1,025 \\
\hline COL. DIST. BUENOS AIRES & 1,0000 & 1,0000 & 0,975 \\
\hline COL. DIST. CAMILO TORRES TENORIO & 0,9532 & 1,0000 & 1,104 \\
\hline COL. DIST. EL CAÑAHUATE & 1,0000 & 1,0000 & 1,013 \\
\hline COL. DIST. HOGAR MARIANO & 0,9955 & 0,9861 & 0,989 \\
\hline COL. DIST. ISAAC NEWTON & 0,9587 & 0,9636 & 0,999 \\
\hline COL. DIST. JORGE ISAACS & 0,9747 & 0,9606 & 0,979 \\
\hline COL. DIST. JOSE EUSEBIO CARO & 0,8834 & 0,9702 & 1,098 \\
\hline COL. DIST. JUAN JOSÉ RONDON & 0,9681 & 0,9427 & 0,974 \\
\hline COLEGIO DISTRITAL LA SALLE & 0,8980 & 0,9084 & 1,012 \\
\hline
\end{tabular}


Continuación Tabla 4. Eficiencia e Índice de Productividad de Malmquist.

\begin{tabular}{|c|c|c|c|}
\hline Institución Educativa & $\begin{array}{l}\text { Eficiencia } \\
\text { CCR } 2012 \\
\end{array}$ & $\begin{array}{l}\text { Eficiencia } \\
\text { CCR } 2013\end{array}$ & $\begin{array}{c}\text { Índice De } \\
\text { Productividad }\end{array}$ \\
\hline COLEGIO DISTRITAL MURILLO & 0,9853 & 0,9445 & 0,923 \\
\hline COL. DIST. NUEVA ESPERANZA & 1,0000 & 1,0000 & 1,040 \\
\hline COL. DIST. NUEVO BOSQUE & 0,9302 & 0,8888 & 0,953 \\
\hline COLEGIO DISTRITAL OLAYA & 0,9664 & 0,9674 & 1,002 \\
\hline COL. DIST. SAGRADO CORAZÓN DE JESÚS & 0,9300 & 0,9231 & 0,989 \\
\hline COL. DIST. SAN GABRIEL & 0,9596 & 1,0000 & 1,061 \\
\hline COL. DIST. SAN JOSÉ & 0,9963 & 0,9867 & 0,991 \\
\hline COL. DIST. SAN VICENTE DE PAUL & 0,9854 & 0,9499 & 0,955 \\
\hline COL. DIST. SANTO DOMINGO DE GUZMÁN & 1,0000 & 1,0000 & 0,997 \\
\hline COLEGIO JORGE N. ABELLO & 0,8434 & 1,0000 & 1,184 \\
\hline COL. MAYOR DE B/QUILLA Y DEL CARIBE & 0,9755 & 0,8942 & 0,917 \\
\hline COL. METROPOLITANO DE B/QUILLA & 0,9891 & 0,9596 & 0,961 \\
\hline COL.TEC. DIST. DE REBOLO & 0,9704 & 0,7674 & 0,758 \\
\hline COL. TEC. DIVERSIFICADO DE B/QUILLA & 0,9530 & 0,8959 & 0,940 \\
\hline ESC. NORMAL SUP. DEL DISTRITO DE B/QUILLA & 0,9850 & 0,9588 & 0,973 \\
\hline ESC. NORMAL SUPERIOR LA HACIENDA & 1,0000 & 1,0000 & 0,999 \\
\hline IED CIUDADELA 20 DE JULIO & 1,0000 & 0,9876 & 0,973 \\
\hline INST.TEC. COMERCIAL EL SANTUARIO & 1,0000 & 1,0000 & 1,019 \\
\hline IED DE FORMACIÓN TEC. ALBERTO ASSA & 1,0000 & 0,9519 & 0,867 \\
\hline INST. CULTURAL LAS MALVINAS & 1,0000 & 0,9904 & 0,963 \\
\hline IED EVARDO TURIZO PALENCIA & 1,0000 & 0,9961 & 0,942 \\
\hline INST. DIST. COM. LOS LAURELES & 1,0000 & 0,9513 & 0,932 \\
\hline IED ARTE Y CULT. ALEJANDRO OBREGÓN & 1,0000 & 1,0000 & 1,109 \\
\hline IED EL CASTILLO DE LA ALBORAYA & 1,0000 & 0,9373 & 0,937 \\
\hline IED NUEVA GRANADA & 1,0000 & 1,0000 & 1,000 \\
\hline IED TEC. MEIRA DEL MAR & 0,9594 & 0,9800 & 1,020 \\
\hline IED BETANIA NORTE & 0,9846 & 1,0000 & 1,016 \\
\hline IED COM. MANUEL ELKIN PATARROYO & 1,0000 & 1,0000 & 0,921 \\
\hline IED DENIS HERRERA DE VILLA & 0,9726 & 0,9981 & 1,006 \\
\hline IED ANTONIO JOSÉ DE SUCRE & 0,9536 & 1,0000 & 1,049 \\
\hline IED ARTE Y TECNOL. ESTHER FORERO & 1,0000 & 0,9884 & 0,948 \\
\hline IED CALIXTO ÁLVAREZ & 0,9710 & 0,9569 & 0,983 \\
\hline IED CARLOS MEISEL & 0,9518 & 0,9191 & 0,943 \\
\hline IED COM. OCTAVIO PAZ & 0,9605 & 0,8960 & 0,925 \\
\hline IED COM. 7 DE ABRIL & 0,9969 & 1,0000 & 0,940 \\
\hline
\end{tabular}


INVESTIGACIONES ANDINA No. 37, Vol. 20

Continuación Tabla 4. Eficiencia e Índice de Productividad de Malmquist.

\begin{tabular}{|c|c|c|c|}
\hline Institución Educativa & $\begin{array}{l}\text { Eficiencia } \\
\text { CCR } 2012\end{array}$ & $\begin{array}{l}\text { Eficiencia } \\
\text { CCR } 2013\end{array}$ & $\begin{array}{c}\text { Índice De } \\
\text { Productividad }\end{array}$ \\
\hline IED COM. METROPOLITANA & 1,0000 & 0,9804 & 0,929 \\
\hline IED CONCENTRACIÓN CEVILLAR & 0,9513 & 0,9336 & 0,975 \\
\hline IED DE FORMACIÓN INTEGRAL & 1,0000 & 1,0000 & 1,000 \\
\hline IED DEL BARRIO SIMÓN BOLIVAR & 0,9429 & 0,8723 & 0,917 \\
\hline IED DEL CARIBE & 1,0000 & 1,0000 & 1,021 \\
\hline IED DEL DLLO HUMANO Y CULT. DEL CARIBE & 0,9665 & 0,9569 & 0,984 \\
\hline IED DESPERTAR DEL SUR & 0,9222 & 0,9613 & 1,014 \\
\hline IED EDUARDO SANTOS & 1,0000 & 1,0000 & 0,953 \\
\hline IED EL CAMPITO & 1,0000 & 0,9845 & 0,971 \\
\hline IED EL PARAÍSO & 0,9946 & 0,9973 & 0,989 \\
\hline IED EL VALLE & 1,0000 & 1,0000 & 0,933 \\
\hline IED EVARISTO SOURDIS & 1,0000 & 1,0000 & 1,133 \\
\hline IED INMACULADA CONCEPCIÓN & 0,9754 & 1,0000 & 1,058 \\
\hline IED INOCENCIO CHINCÁ INEDICH & 0,9896 & 1,0000 & 1,010 \\
\hline IED JESÚS DE NAZARETH & 1,0000 & 0,9735 & 0,953 \\
\hline IED JORGE ROBLEDO ORTIZ & 0,9362 & 0,9620 & 1,027 \\
\hline IED JOSE MARTI & 0,9595 & 1,0000 & 1,034 \\
\hline IED LA CONCEPCIÓN & 1,0000 & 1,0000 & 1,097 \\
\hline IED LA ESMERALDA & 0,9879 & 0,9671 & 0,942 \\
\hline IED LA ESPERANZA DEL SUR & 0,9602 & 0,9724 & 1,008 \\
\hline IED LA LUZ & 0,9565 & 0,9866 & 1,015 \\
\hline IED LA MAGDALENA & 0,9681 & 0,9408 & 0,968 \\
\hline IED LA MILAGROSA FÉ Y ALEGRÍA & 0,9468 & 0,9738 & 1,023 \\
\hline IED LA PRESENTACIÓN & 1,0000 & 1,0000 & 0,977 \\
\hline IED LA VICTORIA & 0,9070 & 0,9002 & 0,992 \\
\hline IED LAS AMÉRICAS & 0,9956 & 1,0000 & 0,955 \\
\hline IED LAS FLORES & 0,9819 & 0,9818 & 0,992 \\
\hline IED LESTONNAC & 1,0000 & 0,9833 & 0,971 \\
\hline IED LUIS CARLOS GALÁN SARMIENTO & 0,8776 & 0,9307 & 1,044 \\
\hline IED LUZ DEL CARIBE & 0,9763 & 0,8884 & 0,910 \\
\hline IED MADRE MARCELINA & 0,9672 & 0,9713 & 1,004 \\
\hline IED MADRES CATÓLICAS & 0,9658 & 0,9322 & 0,965 \\
\hline IED MANUEL ZAPATA OLIVELLA & 1,0000 & 0,9947 & 0,908 \\
\hline IED MARCO FIDEL SUÁREZ & 0,9616 & 0,8929 & 0,924 \\
\hline IED MIGUEL ANGEL BUILES & 0,9679 & 0,9698 & 0,999 \\
\hline
\end{tabular}


Continuación Tabla 4. Eficiencia e Índice de Productividad de Malmquist.

\begin{tabular}{|c|c|c|c|}
\hline Institución Educativa & $\begin{array}{l}\text { Eficiencia } \\
\text { CCR } 2012 \\
\end{array}$ & $\begin{array}{l}\text { Eficiencia } \\
\text { CCR } 2013 \\
\end{array}$ & $\begin{array}{c}\text { Índice De } \\
\text { Productividad }\end{array}$ \\
\hline IED MUNDO BOLIVARIANO & 1,0000 & 1,0000 & 0,890 \\
\hline IED NUESTRA SEÑORA DE LAS NIEVES & 1,0000 & 0,9742 & 0,966 \\
\hline IED PARA EL DLLO DEL TALENTO HUMANO & 0,9927 & 1,0000 & 1,004 \\
\hline IED PARA EL DLLO HUMANO, MARÍA CANO & 0,9930 & 1,0000 & 1,000 \\
\hline INST. EDUC. DIST. PESTALOZZI & 0,9783 & 0,9712 & 0,984 \\
\hline IED REUVEN FEUERSTEIN & 1,0000 & 1,0000 & 1,019 \\
\hline IED SALVADOR SUAREZ SUAREZ & 0,9913 & 1,0000 & 1,003 \\
\hline IED SAN MIGUEL ARCÁNGEL & 0,8995 & 1,0000 & 1,109 \\
\hline IED SAN SALVADOR & 0,9224 & 0,8887 & 0,964 \\
\hline IED SANTA BERNARDITA & 0,9700 & 1,0000 & 1,029 \\
\hline IED SIMON BOLIVAR & 0,9219 & 0,9360 & 1,014 \\
\hline IED TEC. COOP. JESÚS MISERICORDIOSO & 1,0000 & 1,0000 & 0,974 \\
\hline IED TIERRA SANTA & 1,0000 & 1,0000 & 0,994 \\
\hline IED EL CORAZÓN DEL SANTUARIO & 0,9405 & 0,9626 & 1,025 \\
\hline IED RODOLFO LLINÁS RIASCOS & 1,0000 & 0,9491 & 0,941 \\
\hline IED SOFÍA CAMARGO DE LLERAS & 0,9899 & 1,0000 & 1,008 \\
\hline IED TEC. HELENA DE CHAUVIN & 0,9656 & 0,9859 & 1,021 \\
\hline IED CRUZADA SOCIAL & 0,9928 & 1,0000 & 0,993 \\
\hline INST.TEC. DE COMERCIO B/QUILLA & 1,0000 & 1,0000 & 0,998 \\
\hline IED DE LAS NIEVES & 0,9540 & 0,9650 & 1,012 \\
\hline IED BETZABE ESPINOZA & 0,9452 & 0,9575 & 1,007 \\
\hline IED BRISAS DEL RÍO & 1,0000 & 1,0000 & 0,956 \\
\hline IED CIUDADELA ESTUDIANTIL & 1,0000 & 1,0000 & 0,932 \\
\hline IED DE CARRIZAL & 1,0000 & 1,0000 & 0,994 \\
\hline IED EL PUEBLO & 0,9718 & 0,8829 & 0,893 \\
\hline IED FUNDACIÓN PIES DESCALZOS & 0,9836 & 0,9763 & 0,993 \\
\hline IED HILDA MUÑOZ & 0,9950 & 0,9854 & 0,990 \\
\hline IED JAVIER SÁNCHEZ & 0,8047 & 0,9124 & 1,134 \\
\hline IED JUAN ACOSTA SOLERA & 1,0000 & 0,9985 & 0,972 \\
\hline IED KARL PARRISH & 0,9344 & 0,9198 & 0,982 \\
\hline IED LA LIBERTAD & 0,9509 & 0,9350 & 0,981 \\
\hline IED LA MERCED & 0,9903 & 0,9434 & 0,942 \\
\hline IED LA UNIÓN & 0,9390 & 0,9666 & 1,029 \\
\hline IED LAS GRANJAS & 1,0000 & 1,0000 & 0,989 \\
\hline
\end{tabular}


INVESTIGACIONES ANDINA No. 37, Vol. 20

Continuación Tabla 4. Eficiencia e Índice de Productividad de Malmquist

\begin{tabular}{lc|c|c}
\hline \multicolumn{1}{c|}{ Institución Educativa } & $\begin{array}{c}\text { Eficiencia } \\
\text { CCR 2012 }\end{array}$ & $\begin{array}{c}\text { Eficiencia } \\
\text { CCR 2013 }\end{array}$ & $\begin{array}{c}\text { Índice De } \\
\text { Productividad }\end{array}$ \\
\hline IED LOS PINOS & 1,0000 & 1,0000 & 1,029 \\
IED NUESTRA SEÑORA DEL ROSARIO & 1,0000 & 0,9953 & 0,945 \\
IED SANTA MAGDALENA SOFÍA & 0,9756 & 0,9688 & 0,991 \\
IED SONIA AHUMADA & 0,9235 & 0,8240 & 0,894 \\
IED VILLANUEVA & 0,9854 & 1,0000 & 1,005 \\
IED JOSE ANTONIO GALÁN & 0,9898 & 0,9887 & 0,999 \\
INSTITUTO ALEXANDER VON HUMBOLDT & 1,0000 & 1,0000 & 1,014 \\
INST. DIST. DE EXPERIENCIAS PEDAGÓGICAS & 0,9749 & 0,9554 & 0,981 \\
INST. TEC. NACIONAL DE COMERCIO & 0,9776 & 0,9939 & 1,017 \\
\hline
\end{tabular}

Fuente. Elaboración propia de los autores.

La ciudad de Barranquilla se encuentra dividida en cinco localidades; las instituciones educativas están distribuidas en estas zonas de la siguiente manera: 7 IED en la localidad Riomar, 20 IED en la localidad Norte-centro histórico, 32 IED en la localidad Metropolitana, 36 IED en la localidad Suroriente y 53 IED en la localidad Suroccidente.

En la Tabla 5 se muestran los valores de eficiencia promedio por localidad. Los resultados muestran un comportamiento muy similar durante los años 2012 y 2013.

Tabla 5. Eficiencia por Localidad.

\begin{tabular}{c|c|c}
\hline Localidad & $\begin{array}{c}\text { Eficiencia CCR } \\
\mathbf{2 0 1 2}\end{array}$ & $\begin{array}{c}\text { Eficiencia CCR } \\
\mathbf{2 0 1 3}\end{array}$ \\
\hline Localidad Riomar & 0,9784 & 0,9665 \\
Localidad Norte Centro Histórico & 0,9855 & 0,9801 \\
Localidad Metropolitana & 0,9828 & 0,9811 \\
Localidad Suroriente & 0,9672 & 0,9500 \\
Localidad Suroccidente & 0,9671 & 0,9695 \\
\hline
\end{tabular}

22 Fuente. Elaboración propia de los autores. 
Como lo establece la literatura, si la DMU0 obtiene un IPM0 > 1, la unidad ha experimentado una mejora en la productividad con relación al periodo inicial, lo contrario sucede si su IPM0 < 1, y si IPM0 = 1 entonces la DMU0 no ha sufrido ningún cambio productivo. Si vemos los resultados en la Tabla 4 , se aprecia que el IPM mayor lo obtuvo la IED Jorge N Abello, con un IPM=1,184; lo que indica que esta institución experimentó una mejora en su productividad del año 2012 al año 2013. Mientras que el Colegio Técnico Distrital de Rebolo obtuvo un IPM=0,758; por lo que experimentó una disminución en su productividad. Instituciones como el CEB. Y MEDIA \# 176, Col. Dist. Marie Poussepin, IED Nueva Granada, IED de Formación Integral y IED María Cano no sufrieron ningún cambio productivo.

\section{Discusión}

El índice de productividad de Malmquist, junto al análisis envolvente de datos, se han utilizado ampliamente para medir los cambios de productividad en diferentes tipos de organizaciones; sin embargo, su utilización para evaluar el rendimiento del sector educativo es casi nula (20). El presente trabajo es uno de los primeros en Colombia en utilizar el índice de Malmquist en la evaluación de rendimiento de colegios oficiales. En cuanto a estudios de medición de eficiencia en la educación, la gran mayoría de estos hace referencia a instituciones de educación superior, son de carácter regional e involucran un número menor de unidades educa- tivas. En el presente estudio se evaluaron 148 instituciones.

Se observó que durante el año 2012 la eficiencia promedio de los colegios oficiales del distrito de Barranquilla fue de $97,35 \%$ y el número de instituciones eficientes fue 54, lo cual equivale al $36 \%$ del total. Para el año 2013, la eficiencia promedio de los colegios oficiales del distrito de Barranquilla fue de 96,86\% y el número de instituciones eficientes fue 57 , lo cual equivale al $38 \%$ del total. De acuerdo con lo anterior, las IED podrían lograr los mismos resultados con un ahorro potencial de los insumos empleados del 2,65\% y 3,14\% en 2012 y 2013. Durante este periodo, 37 instituciones no presentaron cambio en sus valores de eficiencia. Los resultados indican que, en términos de eficiencia, el comportamiento de las IED del distrito de Barranquilla es muy similar, ya que las que no son eficientes muestran resultados superiores al $80 \%$ y al $77 \%$ para el 2012 y 2013, respectivamente. Los resultados no muestran diferencias significativas en la eficiencia promedio por localidades.

Los resultados ponen de manifiesto que la productividad del sector de la IED del distrito de Barranquilla sufre un leve descenso del 1,4\%. Sin embargo, se puede observar que los mejores colegios en este ítem logran un aumento del $4,4 \%$ en promedio y los peores colegios una disminución del 3,5\% en promedio, lo que evidencia que estos últimos no han sido capaces en términos medio de adoptar mejores técnicas organizativas para incrementar su productividad. 


\section{Conclusiones}

Este estudio estuvo orientado a evaluar y analizar la eficiencia y la productividad de todas las instituciones educativas del distrito de Barranquilla durante el periodo 2012-2013, la selección de los insumos o variables de entrada y de los productos o variables de salidas representaba una tarea de mucho cuidado, ya que la calidad de los resultados estaba sujeta a una buena selección de estas variables. Por este motivo, se inició un proceso exhaustivo para identificar los recursos y productos que caracterizaban el sector educativo oficial de la ciudad de Barranquilla. Se consultaron los trabajos empíricos en esta área, que eran comparables para definir

\section{Referencias}

1. Golany B., Roll Y. An application proedure for DEA. Omega. 1989;17(3):237-50.

2. Congreso de la República. Por la cual se expide la Ley General de Educación. Ley 115. Colombia: Ministerio de Educación; 1994. 50 p.

3. Banco Mundial. [Internet]. Gasto público en educación total (\% del PIB). S.F. [Citado año, mes, día]. Disponible en: http:// datos.bancomundial.org/indicador/SE.XPD.TOTL.GD.ZS

4. Charnes A., Cooper W., Rhodes E. Measuring the efficiency of decision-making units. Eur J Oper Res. 1978;2(6):429-44.

5. Guzmán-Finol., K. ¿Qué hay detrás de un cambio en la productividad hospitalaria? Documentos de Trabajo sobre Economía Regional. 2015;(215):1-56. inicialmente el grupo de variables, para luego validarlas con personas con experiencias y formación en el área de la educación.

La eficiencia de las IED durante los años 2012 y 2013 es muy similar. De las 148 IED, durante los años 2012 y 2013 resultaron eficientes 54 IED y 57 IED respectivamente. Se encontró una disminución de la productividad del sector; sin embargo, 56 instituciones presentaron un IPM0 > 1, así: 1 IED de la localidad Riomar, 10 IED de la localidad Norte-centro histórico, 9 IED de la localidad Metropolitana, 12 IED de la localidad Suroriente y 24 de localidad Suroccidente. La localidad Norte-centro histórico fue la única que presentó incremento de productividad.

6. Kutlar A., Kabasakal A., Ekici M. Efficiency of commercial banks in Turkey and their comparison: application of DEA with Tobit analysis. International Journal of Mathematics in Operational Research. 2017;10(1):84-103.

7. Tsolas I. Firm credit risk evaluation: a series two-stage DEA modeling framework. Ann of Oper Res. 2015;233(1):483-500.

8. Carrillo M., Jorge J. DEA-like efficiency ranking of regional health systems in Spain. Soc Indic Res. 2017;133(3):1133-49.

9. Sav T. Efficiency Estimates and Rankings Employing Data Envelopment and Stochastic Frontier Analyses: Evaluating the Management of U.S. Public Colleges. Information Management and Business Review. 2012;8(4):444-52. 
10. Nazarko J., Šaparauskas J. Application of DEA method in efficiency evaluation of public higher education institutions. Technological and Economic Development of Economy. 2014;20(1):25-44.

11. Liu HH., Kuo FH., Li LH. The operating efficiency of vocational and senior high schools in Xindian district of New Taipei City: Three envelopment models in DEA. International Business Research. 2016;9(11):116-25

12. Garzón H., Flores S., Flores J. Eficiencia técnica de instituciones públicas de educación secundaria del estado Barinas, Venezuela. Revista Venezolana de Análisis de Coyuntura. 2011;17(2):149-65.

13. Quesada V., Blanco I., Maza F. Análisis envolvente de datos aplicado a la cobertura educativa en el departamento de Bolívar, Colombia (2007-2008). Omnia. 2010;16(3):77-100.

14. Iregui A., Melo L., Ramos J. Análisis de eficiencia de la educación en Colombia. Rev de Economía del Rosario. 2007;10(1):21-41.

15. Soto J., Vásquez S., Villegas J. Eficiencia en cobertura y calidad de las instituciones educativas oficiales del municipio de Dosquebradas (Risaralda). Scientia Et Technica. 2010; 3(46):181-6.

16. Caves D., Christensen L., Diewert E. The economic theory of index numbers and the measurement of input, output and productivity. Econométrica. 1982;50(6):1393-1414.

17. Malmquist S. Index numbers and indifference surfaces. Trabajos de Estadística y de Investigación Operativa.1953;4(2):209-242.

18. Aparicio J., Crespo-Cebada E., Pedraja-Chaparro F., Santín D. Comparing school ownership performance using a pseudo-panel database: a Malmquist-type index approach. Eur J Oper Res. 2017;256(2):533-42.
19. Podinovski V., Ismail I., Bouzdine-Chameeva T., Zhang W. Combining the assumptions of variable and constant returns to scale in the efficiency evaluation of secondary schools. Eur J Oper Res. 2014;239(2):504-13.

20. Essid H., Ouellette P., Vigeant S. Productivity, efficiency, and technical change of Tunisian schools: A bootstrapped Malmquist approach with quasi-fixed inputs. Omega. 2014;42(1), 88-97.

21. Cooper W., Seiford L., Tone K. Data envelopment analysis. 2da ed. Nueva York: Springer Science \& Business Media;2007. $492 \mathrm{p}$.

22. Escobar J., Cuervo A. Validez de contenido y juicio de expertos: una aproximación a su utilización. Avances en Medición. 2008;6:27-36.

23. Jenkins L., Anderson M. A multivariate statistical approach to reducing the number of variables in data envelopment analysis. Eur Journal Oper Res. 2003;147(1):51-61.

24. Sistema de información nacional de educación básica y media (SINEB) [Internet]. http://www.mineducacion.gov. co/1621/w3-article-156285.html.

25. Departamento Administrativo de la Función Pública. Por el cual se modifica la remuneración de los servidores públicos docentes y directivos docentes al servicio del Estado en los niveles de preescolar, básica y media que se rigen por el Decreto Ley 2277 de 1979. Decreto 1055. Colombia: Ministerio de Educación. 2011. 7 p.

26. Departamento Administrativo de la Función Pública. Por el cual se modifica la remuneración de los servidores públicos docentes y directivos docentes al servicio del Estado en los niveles de preescolar, básica y media que se rigen por el Decreto Ley 1278 de 2002. Decreto 1027. Colombia: Ministerio de Educación. 2011. 7 p. 
27. Suzuki S., Nijkamp P., Rietveld P., Pels E. A distance friction minimization approach in data envelopment analysis: a comparative study on airport efficiency. Eur $\mathrm{J}$ of Oper Res. 2010;207(2):1104-15.

28. Färe R., Grosskopf S., Lindgren B., Roos P. Productivity changes in Swedish pharmacies 1980-1989: a non-parametric Malmquist approach. Journal of Productivity Analysis. 1992;3(1-2):85-101.

29. Parteka A., Wolszczak-Derlacz J. Dynamics of productivity in higher education: cross-european evidence based on bootstrapped Malmquist indices. Journal of Productivity Analysis. 2013;40(1):67-82. 